\title{
The Immediate and Short Term Impact of Successful Percutaneous Transvenous Mitral Commissurotomy on Right Ventricular Function
}

\section{Santosh Kumar C, Rajasekhar D*, Vanajakshamma V and Boochi Babu M}

Department of Cardiology, SVIMS, Tirupati, Andhra Pradesh, India

\begin{abstract}
Objectives: The purpose of this study was to evaluate the immediate and short term follow up impact of Percutaneous Transvenous Mitral Commissurotomy (PTMC) on Right Ventricular (RV) function using twodimensional and tissue Doppler echocardiographic indices.

Background: In patients with Mitral Stenosis (MS) RV function may be affected due to myocardial and hemodynamic factors. Previous studies using echocardiography have shown discordant results as regards to improvement of RV function immediately after PTMC. Only few studies have evaluated RV function at 6 months follow up.

Methods: A total of 90 patients with severe symptomatic MS, all in sinus rhythm, who got admitted for PTMC to author's institute, were prospectively enrolled. RV function was evaluated by conventional and Tissue Doppler Imaging (TDI) echocardiography before, 48 hours after PTMC, and at 6 months follow up.

Results: All patients underwent successful PTMC. The mitral valve area was significantly increased $(0.80 \pm$ $0.13 \mathrm{~cm}^{2}$ versus $\left.1.73 \pm 0.14 \mathrm{~cm}^{2}, \mathrm{p}=0.0001\right)$ immediately after PTMC, while the mean transmitral gradient $(21.6 \pm$ $8.0 \mathrm{~mm} \mathrm{Hg}$ versus $5.9 \pm 1.6 \mathrm{~mm} \mathrm{Hg}, \mathrm{p}<0.0001)$ and systolic pulmonary artery pressure $(52.3 \pm 23.4 \mathrm{~mm} \mathrm{Hg}$ versus $41.7 \pm 16.9 \mathrm{~mm} \mathrm{Hg}, \mathrm{p}<0.0001$ ) were significantly decreased. There was significant improvement in RV function immediately after PTMC (RV outflow tract fractional shortening (RVOTfs): $54.1 \pm 8.7 \%$ versus $70.4 \pm 5.0 \%, p<$ 0.001, Tricuspid Annular Plane Systolic Excursion (TAPSE): $16.0 \pm 1.5 \mathrm{~mm}$ versus $18.6 \pm 1.7 \mathrm{~mm}, \mathrm{p}<0.0001$, TEl INDEX: $0.51 \pm 0.04$ to $0.34 \pm 0.04, p<0.001$ ). Myocardial velocities measured at lateral tricuspid annulus were not improved significantly immediately after PTMC but improved significantly at 6 months follow up (Myocardial velocity during isovolumic contraction (IVCv); $10.2 \pm 0.6 \mathrm{~cm} / \mathrm{s}$ to $12.0 \pm 1.3 \mathrm{~cm} / \mathrm{s}, \mathrm{p}<0.001$, systolic myocardial velocity (Sv): $11.7 \pm 0.9 \mathrm{~cm} / \mathrm{s}$ to $13.2 \pm 0.9 \mathrm{~cm} / \mathrm{s}, \mathrm{p}<0.001$, myocardial acceleration during isovolumic contraction(IVA): $3.0 \pm 0.5$ $\mathrm{cm} / \mathrm{s}^{2}$ to $3.9 \pm 0.3 \mathrm{~cm} / \mathrm{s}^{2}, \mathrm{p}<0.001$ ).
\end{abstract}

Conclusion: Immediately after successful PTMC, significant improvement in RV function was observed. TDI myocardial velocities and IVA showed gradual improvement in RV function at 6 months follow up. Prognostic value and clinical significance of this improvement deserve further investigation.

Keywords: Mitral stenosis; Rheumatic heart disease; Doppler tissue imaging

\section{Introduction}

Rheumatic heart disease causes significant morbidity and mortality. Mitral stenosis (MS) is the commonest presentation in rheumatic heart disease. Rheumatic MS is a frequent cause of valve disease in developing countries [1]. Despite the striking decrease in the prevalence of rheumatic fever in western countries it still accounts for $12 \%$ of native valvular heart disease [2]. The treatment option and its timing should be decided on the basis of clinical, morphological, and functional characteristics. Since its introduction in 1984 by Inoue et al. [3] Percutaneous Transvenous Mitral Commissurotomy (PTMC) has become established as a safe and effective treatment for rheumatic MS and remains the treatment of choice in patients with a favorable anatomy [4-6].

The Right Ventricular (RV) function is an important determinant of clinical symptoms, exercise capacity, pre-operative survival and postoperative outcome in patients with MS [7]. In patients with MS, the $\mathrm{RV}$ function may be altered due to an increase in the left atrial pressure and/or changes in the pulmonary arteriolar vasculature or may be affected by the rheumatic process directly [8]. The results of previous studies, using either invasive or radionuclide methods, demonstrated long-term improvement in RV function after PTMC $[9,10]$.

In patients with MS, previous studies have shown discordant results as regards to improvement of Right Ventricular (RV) function immediately after PTMC and only few studies [11-13] have evaluated the RV function during follow up. Hence, the purpose of this study was to evaluate the immediate and short term follow up impact of PTMC on RV function using two-dimensional and tissue Doppler echocardiographic indices.

\section{Materials and Methods}

The study population consisted of 90 patients with symptomatic MS who underwent PTMC in cardiology department of Sri Venkateswara Institute of Medical sciences, Tirupati between August 2011 and April 2012. Patients in all age groups, with evidence of severe MS \{Mitral

*Corresponding author: D. Rajasekhar, Professor and Head, Department of Cardiology, SVIMS, Tirupati-517507, Andhra Pradesh, India, Tel: +91-9849221650; E-mail: cardiologysvims@gmail.com

Received May 05, 2015; Accepted July 30, 2015; Published August 03, 2015

Citation: Santosh Kumar C, Rajasekhar D, Vanajakshamma V, Boochi Babu M (2015) The Immediate and Short Term Impact of Successful Percutaneous Transvenous Mitral Commissurotomy on Right Ventricular Function. J Cardiovasc Dis Diagn 3: 217. doi:10.4172/2329-9517.1000217

Copyright: (c) 2015 Santosh Kumar C, et al. This is an open-access article distributed under the terms of the Creative Commons Attribution License, which permits unrestricted use, distribution, and reproduction in any medium, provided the original author and source are credited. 
Citation: Santosh Kumar C, Rajasekhar D, Vanajakshamma V, Boochi Babu M (2015) The Immediate and Short Term Impact of Successful Percutaneous Transvenous Mitral Commissurotomy on Right Ventricular Function. J Cardiovasc Dis Diagn 3: 217. doi:10.4172/23299517.1000217

Page 2 of 7

Valve Area $\left(\right.$ MVA) $\left.<1.0 \mathrm{~cm}^{2}\right\}$ admitted in our institution, in whom PTMC was feasible were included. Those who were fulfilling the PTMC intervention criteria and those who had experienced a successful intervention only were included. All participants gave their informed consent. Institutional Ethical Committee clearance was obtained for conducting the study.

\section{Exclusion criteria}

1. Atrial fibrillation

2. Systemic hypertension

3. Diabetes mellitus

4. More than mild mitral or aortic regurgitation and/or aortic stenosis, organic pulmonary and tricuspid valve disease

5. New York Heart Association functional class IV

6. Patients with lung disease

7. Pregnancy.

\section{Echocardiographic measurements}

All patients underwent Two-dimensional (2D) echocardiography and Doppler Tissue Imaging (DTI) studies before PTMC, 48 hours after PTMC and at 6 months follow up. All studies were obtained using a Philips IE-33 ultrasonographic machine equipped with a $3.5 \mathrm{MHz}$ transducer.

\section{Evaluation of MS severity and general parameters}

Mitral Valve Area (MVA) was determined by planimetry in every patient. The peak and mean mitral valve transmitral pressure gradients were measured using the Bernoulli principle from continuous wave Doppler recordings through the center of mitral inflow [5].

The Wilkins score was used to judge mitral leaflet mobility, valvular and subvalvular thickening, and calcification. Forty eight hours after mitral balloon dilatation and at 6 months follow up; MVA was again determined by planimetry. Systolic Pulmonary Artery Pressure (SPAP) was derived from the tricuspid regurgitant jet peak velocity using the modified Bernoulli equation (peak gradient $4 \mathrm{~V}^{2}+$ mean right atrial pressure, where $\mathrm{V}$ is the maximal velocity of the tricuspid regurgitant jet) (Right atrial pressure is calculated by estimating the inferior vena caval size and it's variation with respiration) [14].

\section{Right ventricular function assessment}

All echocardiographic parameters of RV function were measured according to guidelines for echocardiographic assessment of the right heart in adults [14]. From the parasternal short-axis view at the level of the aortic root, the RV outflow tract diameters at end-diastole and end-systole were measured. RV Outflow Tract Fractional Shortening (RVOTfs) was calculated using the formula [14].

RVOTfs $=($ RVOTd - RVOTs $) /$ RVOTd where RVOTd and RVOTs represent end-diastolic and end-systolic dimensions of RVOT.

The Tricuspid Annular Plane Systolic Excursion (TAPSE) was determined by the difference in the displacement of the RV base during systole and diastole [15] (Figure 1). RV end-diastolic and end-systolic areas were measured from the apical four chamber view to calculate RV Fractional Area Change (RVFAC) [16,17]. With the same views, the
RV ejection fraction was calculated using Simpson's rule [14].

\section{Pulsed wave DTI}

The Tei index of RV myocardial performance was calculated as the time between tricuspid valve closure to tricuspid valve opening, divided by the RV ejection time, determined by pulsed Doppler [18] (Figure 2). A $3.5 \mathrm{~mm}$ sample volume was placed at the septal and lateral side of the tricuspid annulus. Peak myocardial velocities during systole, early, and late diastole were measured at a sweep speed of $100 \mathrm{~mm} / \mathrm{s}$ [18,19] (Figure 3).

Myocardial acceleration during Isovolumic Contraction (IVA) was measured by dividing myocardial velocity during isovolumic contraction by the time interval from onset of the myocardial velocity during isovolumic contraction to the time at peak velocity of this wave [20]. The final values of all parameters were obtained after averaging over

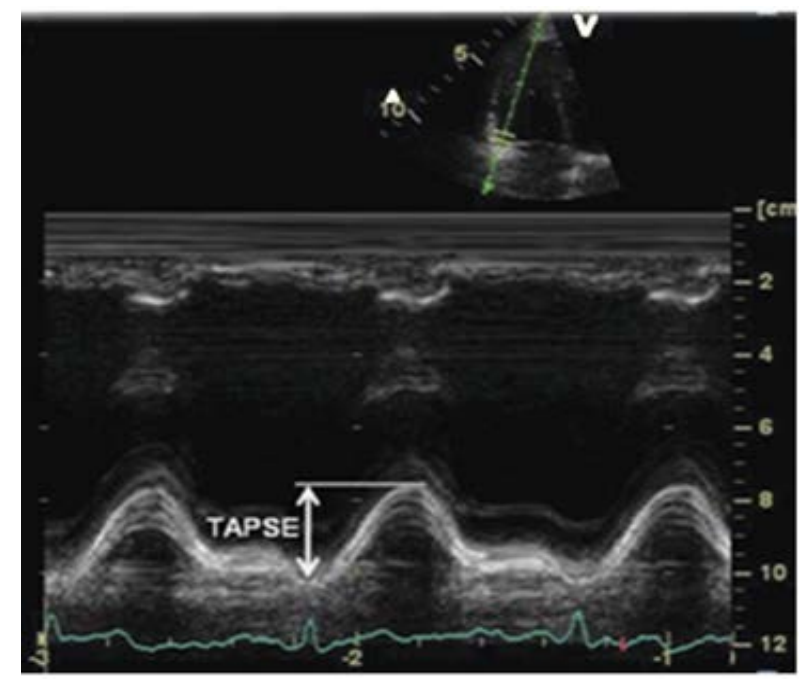

Figure 1: Measurement of tricuspid annular plane systolic excursion (TAPSE).

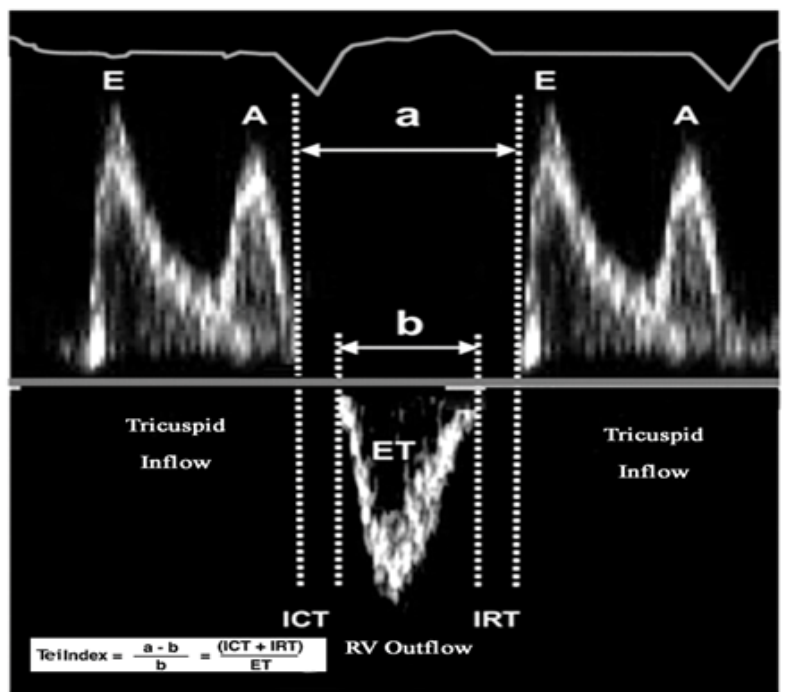

Figure 2: Measurement of right ventricular Ejection Time (ET), Isovolumetric Contraction Time (ICT), Isovolumetric Relaxation Time (IRT), and calculation of Tei Index. 
Citation: Santosh Kumar C, Rajasekhar D, Vanajakshamma V, Boochi Babu M (2015) The Immediate and Short Term Impact of Successful Percutaneous Transvenous Mitral Commissurotomy on Right Ventricular Function. J Cardiovasc Dis Diagn 3: 217. doi:10.4172/23299517.1000217

Page 3 of 7

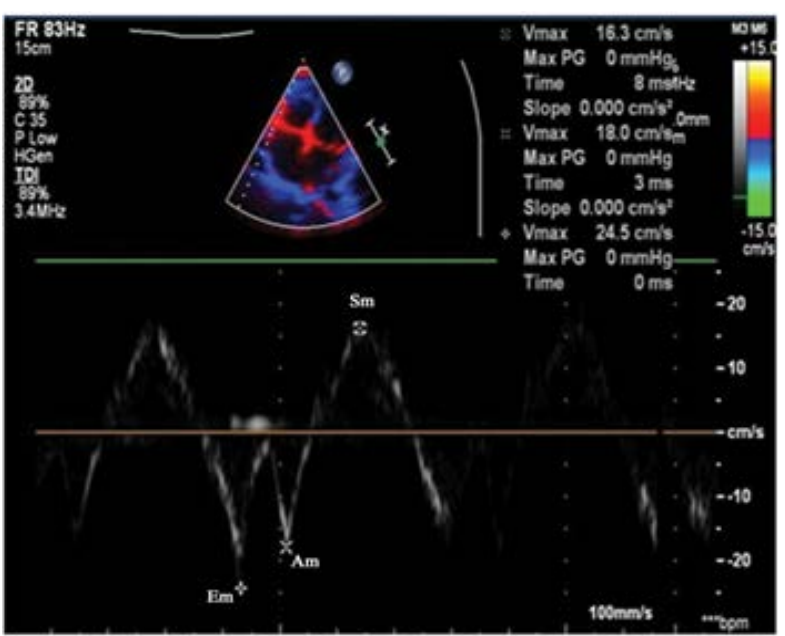

Figure 3: Measurement of lateral tricuspid annular tissue Doppler velocities.

three cardiac cycles. All measurements were recorded at pre-PTMC, postPTMC and at 6 month follow up.

\section{Percutaneous transvenous mitral commissurotomy}

The PTMC was performed via an antegrade transvenous approach using an Inoue balloon and stepwise dilatation strategy [3]. The nominal balloon diameter was decided according to the height of the patient (i.e. height $(\mathrm{cm}) / 10+10=$ balloon diameter). Successful PTMC was defined as either post commissurotomy mitral valve area (MVA) $>1.5 \mathrm{~cm}^{2}$ or a MVA of more than twice the pre-procedural value, together with no worsening of mitral regurgitation $>$ grade $2+$.

\section{Statistical analysis}

Statistical analysis was performed with SPSS version 20.0. Data was presented as mean \pm SD for continuous variables and as percentages for categorical variables. Kolmogorov-smirnov test of normality was performed to check the normal distribution. Comparisons of values before and after PTMC were performed using paired sample t-test. A p value less than 0.05 was considered to indicate statistical significance.

\section{Results}

\section{Demographic characteristics of the patients}

Ninety patients with MS in sinus rhythm were enrolled prospectively into the study. All patients had severe MS and were symptomatic, while
10 patients had a history of previous commissurotomy. The clinical and 2D echocardiographic characteristics of patients are summarized in Tables 1 and 2, respectively.

\section{D echocardiographic parameters before and after PTMC}

Following PTMC, the 2D MVA increased from $0.80 \pm 0.13 \mathrm{~cm}^{2}$ to $1.73 \pm 0.14 \mathrm{~cm}^{2}(\mathrm{p}=0.0001)$. Peak transmitral gradients and mean transmitral gradients before PTMC were $33.0 \pm 10.2 \mathrm{~mm} \mathrm{Hg}$ and 21.6 $\pm 8.0 \mathrm{~mm} \mathrm{Hg}$, and significantly decreased after PTMC to $11.0 \pm 2.5 \mathrm{~mm}$ $\mathrm{Hg}$ and $5.9 \pm 1.6 \mathrm{~mm} \mathrm{Hg}(\mathrm{p}<0.0001$ and $<0.0001)$ respectively.

A significant fall in systolic pulmonary artery pressure (SPAP) was seen immediately after PTMC from $52.3 \pm 23.4 \mathrm{~mm} \mathrm{Hg}$ to $41.7 \pm 16.9$ $\mathrm{mm} \mathrm{Hg}(\mathrm{p}<0.0001)$. A further fall in SPAP was observed at 6 months follow-up $(38.1 \pm 16.7)$. LA diameter was $44.9 \pm 5.9 \mathrm{~mm}$ prior to PTMC, significantly decreased to $42.4 \pm 6.0 \mathrm{~mm}(\mathrm{p}=0.006)$ after PTMC. LV Ejection Fraction (LVEF), LV End-Diastolic Diameter (LVEDD) and LV End-Systolic Diameter (LVESD) measurements were unchanged after PTMC.

\section{D echocardiographic assessment of RV function}

Mean RV Ejection Fraction (RVEF) of patients before PTMC was $50.0 \pm 6.9 \%$, did not change significantly immediately after PTMC. But there was a significant improvement in RVEF seen at 6 months follow-

\begin{tabular}{|c|c|}
\hline Characteristics & \\
\hline Age 33.6 $( \pm 9.8)$ yrs & Age 33.6 $( \pm 9.8)$ yrs. \\
\hline Sex & $62(69 \%)$ \\
Male & $28(31 \%)$ \\
Female & 0 \\
\hline Functional class (NYHA) & $36(40 \%)$ \\
Class I & $54(60 \%)$ \\
Class II & 0 \\
Class III & $10(11 \%)$ \\
\hline Class IV & $2(2.2 \%)$ \\
\hline Previous PTMC & 0 \\
\hline Previous CMV & 0 \\
\hline CVA History & $155.7 \pm 9.3 \mathrm{~cm}$ \\
\hline Atrial fibrillation & $44.9 \pm 5.9 \mathrm{~mm}$ \\
\hline Height & $3(3.3 \%)$ \\
\hline LA size & $60.01 \pm 5.8 \%$ \\
\hline Left ventricular dysfunction & $6.8 \pm 1.0$ \\
\hline Left ventricular ejection fraction & \\
\hline Wilkins score & \\
\hline
\end{tabular}

CVA: Cerebro Vascular Accident; CMV: Closed Mitral Valvotomy; NYHA: New York Heart Association; LA: Left Atrium; PTMC: Percutaneous Transvenous Mitral Commissurotomy.

Table 1: Baseline characteristics of the population.

\begin{tabular}{|c|c|c|c|c|c|}
\hline Variable & Pre PTMC (A) & Post PTMC (B) & $p$ value (A Vs $B$ ) & follow-up (C) & $p$ value (A Vs $C)$ \\
\hline $\mathrm{LA}, \mathrm{mm}$ & $44.9 \pm 5.9$ & $42.4 \pm 6.0$ & 0.006 & $40.3 \pm 5.9$ & $<0.001$ \\
\hline LVEF, \% & $60.0 \pm 5.8$ & $60.2 \pm 5.7$ & NS & $59.6 \pm 8.2$ & NS \\
\hline LV diastolic diameter, $\mathrm{mm}$ & $41.1 \pm 5.1$ & $41.4 \pm 5.1$ & NS & $42.2 \pm 5.8$ & NS \\
\hline LV systolic diameter, mm & $27.2 \pm 4.2$ & $27.3 \pm 4.5$ & NS & $27.8 \pm 5.1$ & NS \\
\hline Max Gradient, mm Hg & $33.0 \pm 10.2$ & $11.0 \pm 2.5$ & $<0.001$ & $9.8 \pm 2.7$ & $<0.001$ \\
\hline Mean Gradient, $\mathrm{mm} \mathrm{Hg}$ & $21.6 \pm 8.0$ & $5.9 \pm 1.6$ & $<0.001$ & $5.6 \pm 1.5$ & $<0.001$ \\
\hline MVA PHT, $\mathrm{cm} 2$ & $0.84 \pm 0.13$ & $1.87 \pm 0.14$ & $<0.001$ & $1.84 \pm 0.21$ & $<0.001$ \\
\hline MVA planimetry, cm2 & $0.80 \pm 0.13$ & $1.73 \pm 0.14$ & $<0.001$ & $1.73 \pm 0.14$ & $<0.001$ \\
\hline PASP, mm Hg & $52.3 \pm 23.4$ & $41.7 \pm 16.9$ & $<0.001$ & $38.1 \pm 16.7$ & $<0.001$ \\
\hline
\end{tabular}

LVEF: Left ventricular ejection fraction; LA: left atrial diameter; LV: left ventricular; Max Gr, transmitral Maximum gradient; Mean Gr: transmitral mean gradient; MVA PHT: pressure half-time mitral valve area; MVA plan: planimetric mitral valve area; NS: statistically not significant; PASP: pulmonary artery Systolic pressure; (A Vs B), Pre PTMC Values compared with immediate post PTMC values; (A Vs C), Pre PTMC values compared with 6 months follow-up values. 
Citation: Santosh Kumar C, Rajasekhar D, Vanajakshamma V, Boochi Babu M (2015) The Immediate and Short Term Impact of Successful Percutaneous Transvenous Mitral Commissurotomy on Right Ventricular Function. J Cardiovasc Dis Diagn 3: 217. doi:10.4172/23299517.1000217

Page 4 of 7

up (52.9. $\pm 6.0 \%, \mathrm{p}=0.003)$. RV Fractional Area Change (RVFAC) before PTMC was $34.4 \pm 4.3 \%$, did not change significantly immediately after PTMC, but significant improvement was noted at 6 months follow up visit $(36.0 \pm 4.7 \%, \mathrm{p}<0.001)$, shown in (Table 3 ).

RV TEI index decreased significantly from $0.51 \pm 0.04$, to $0.34 \pm$ 0.04 after PTMC, no further change was noted at 6 months follow up. Both RVOT Fractional Shortening (RVOTfs) and Tricuspid annular plane systolic excursion (TAPSE) were $54.1 \pm 8.7 \%$ and $16.0 \pm 1.5$ $\mathrm{mm}$, respectively prior to PTMC. Both these parameters increased significantly immediately after PTMC to $70.4 \pm 5.0 \%$ and $18.6 \pm 1.7$ $\mathrm{mm}$. At 6 month follow up both these variables further improved to $74.1 \pm 5.5 \%$ and $19.9 \pm 2 \mathrm{~mm}$ (Table 3 ).

\section{Tissue doppler imaging variables of RV function}

Both myocardial acceleration during Isovolumic Contraction (IVA) and myocardial velocity during Isovolumic Contraction (IVCV) measured at lateral tricuspid annulus did not change significantly immediately after PTMC ( $\mathrm{p}=0.1$ and 0.09 respectively), but increased significantly when measured at 6 months after PTMC (Table 4). Systolic myocardial velocities (Sv) measured at lateral tricuspid annulus did not change immediately after PTMC but increased significantly at 6 month follow up period (Table 4).

Early and late diastolic myocardial velocities measured at lateral tricuspid annulus did not change after PTMC, both immediately and at 6 months follow up.

\section{Correlation between MVA by planimetry and other parameters before PTMC}

Table 5 showed significant correlation between MVA by planimetry with (MVA by PHT, mean pressure and SPAP) and no relation with the other parameters. Comparison of pre- and post-PTMC (at 48 hours and 6 months) echocardiographic variables in patients with or without baseline pulmonary hypertension (Table 6).

\begin{tabular}{|c|c|c|c|c|c|}
\hline Variable & $\begin{array}{c}\text { Pre PTMC } \\
\text { (A) }\end{array}$ & $\begin{array}{c}\text { Post PTMC } \\
\text { (B) }\end{array}$ & $\begin{array}{c}\text { p value } \\
\text { (A Vs B) }\end{array}$ & $\begin{array}{c}\text { follow-up } \\
\text { (C) }\end{array}$ & $\begin{array}{c}\text { p value } \\
\text { (A Vs C) }\end{array}$ \\
\hline RV EF, \% & $50.0 \pm 6.9$ & $50.9 \pm 6.0$ & NS & $52.9 \pm 6.0$ & $=0.003$ \\
\hline RVFAC, \% & $34.4 \pm 4.3$ & $35.1 \pm 4.7$ & NS & $36.0 \pm 4.7$ & $<0.001$ \\
\hline RVOTfs, $\%$ & $54.1 \pm 8.7$ & $70.4 \pm 5.0$ & $<0.001$ & $74.1 \pm 5.5$ & $<0.001$ \\
\hline TAPSE, mm & $16.0 \pm 1.5$ & $18.6 \pm 1.7$ & $<0.001$ & $19.9 \pm 2.6$ & $<0.001$ \\
\hline TEI INDEX & $0.51 \pm 0.04$ & $0.34 \pm 0.04$ & $<0.001$ & $0.32 \pm 0.04$ & $<0.001$ \\
\hline
\end{tabular}

RVEF: RV Ejection Fraction; RVFAC: RV fractional area change; RVOTfs: RV outflow tract fractional Shortening; TAPSE: Tricuspid Annular Plane Systolic Excursion; (A Vs B), Pre PTMC Values compared with immediate post PTMC values; (A Vs C), Pre PTMC values compared with 6 months follow-up values.

Table 3: Comparison of $2 \mathrm{D}$ echocardiographic parameters of RV function before and after PTMC.

\begin{tabular}{|c|c|c|c|c|c|}
\hline Variable & $\begin{array}{c}\text { Pre PTMC } \\
\text { (A) }\end{array}$ & $\begin{array}{c}\text { Post PTMC } \\
\text { (B) }\end{array}$ & $\begin{array}{c}\text { p value } \\
\text { (A Vs B) }\end{array}$ & $\begin{array}{c}\text { follow-up } \\
\text { (C) }\end{array}$ & $\begin{array}{c}\text { p value } \\
\text { (A Vs C) }\end{array}$ \\
\hline Lateral EV CM/S & $13.7 \pm 0.8$ & $14.1 \pm 1.8$ & 0.22 & $14.4 \pm 1.3$ & 0.08 \\
\hline Lateral AV CM/S & $13.3 \pm 0.6$ & $13.8 \pm 1.6$ & 0.34 & $14.2 \pm 0.8$ & 0.1 \\
\hline Lateral SV CM/S & $11.7 \pm 0.9$ & $11.9 \pm 1.3$ & 0.08 & $13.2 \pm 0.9$ & $<0.001$ \\
\hline Lateral IVC V CM/S & $10.2 \pm 0.6$ & $10.5 \pm 0.9$ & 0.09 & $12.0 \pm 1.3$ & $<0.001$ \\
\hline Lateral IVA CM/S & $3.0 \pm 0.5$ & $3.1 \pm 0.5$ & 0.1 & $3.9 \pm 0.3$ & $<0.001$ \\
\hline
\end{tabular}

RV: Right Ventricle; TDI: Tissue Doppler Imaging; PTMC: Percutaneous Transvenous Mitral Commissurotomy; IVCv: Myocardial velocity during isovolumic contraction; IVA: Isovolumetric Velocity Acceleration; Ev: E wave velocity; Av: A wave velocity; Sv: Systolic myocardial velocity

Table 4: RV TDI myocardial velocities of lateral tricuspid annulus.

\begin{tabular}{|c|c|c|}
\hline & \multicolumn{3}{|c|}{ MVA by planimetry } \\
\hline MVA PHT & r-value & p-value \\
\hline Max Gr & 0.436 & $<0.0001$ \\
\hline Mean Gr & -0.201 & 0.057 \\
\hline PASP & -0.430 & $<0.0001$ \\
\hline RVOTfs & -0.229 & 0.030 \\
\hline RVFAC & -0.104 & 0.330 \\
\hline RV TEI INDEX & 0.047 & 0.660 \\
\hline TAPSE & -0.115 & 0.280 \\
\hline RV EF & 0.107 & 0.316 \\
\hline Lateral E' & 0.050 & 0.639 \\
\hline Lateral A' & 0.053 & 0.623 \\
\hline Lateral Sv & 0.060 & 0.576 \\
\hline Lateral IVCv & 0.005 & 0.962 \\
\hline Lateral IVA & 0.005 & 0.965 \\
\hline WILKINS Score & 0.024 & 0.825 \\
\hline
\end{tabular}

MVA: Mitral Valve Area; PTMC: Percutaneous Transvenous Mitral Commissurotomy MVA PHT: Pressure Half-Time Mitral Valve Area; Max Gr: Transmitral Maximum Gradient; Mean Gr: Transmitral Mean Gradient; PASP: Pulmonary Artery Systolic Pressure; RVOTfs: RV Outflow Tract fractional shortening, RVFAC: RV Fractional Area Change; TAPSE: Tricuspid Annular Plane Systolic Excursion; RVEF; RV Ejection Fraction, Ev: E wave velocity; Av: A wave velocity; Sv: Systolic myocardial velocity; IVCv: Myocardial velocity during isovolumic contraction; IVA: Isovolumetric Velocity Acceleration.

Table 5: Correlation between MVA by planimetry and other parameters before PTMC.

There was significant fall in SPAP in both pulmonary hypertensive and non-pulmonary hypertensive group immediately after PTMC and after 6 months. Various two-dimensional echocardiographic parameters of RV function and DTI findings were represented in Table 6.

\section{Discussion}

In patients with MS, RV function is closely related to symptoms, functional capacity, need and timing for interventions, perioperative mortality, and postoperative results [7]. Evaluation of RV function by conventional transthoracic echocardiography is difficult due to its asymmetrical shape, narrow acoustic window, and geometrical assumptions for calculation of volumes. Quantitative echocardiographic assessment of the RV is difficult, a wide variety of techniques have been proposed but none of the echocardiographic indices is considered gold standard at present. We studied various two-dimensional echocardiographic and Doppler Tissue Imaging (DTI) parameters to assess the RV function in patients with MS immediately after successful PTMC and at 6 months follow-up period.

\section{DTI of lateral tricuspid annulus}

DTI echocardiography permits assessment of longitudinal RV function by measuring systolic myocardial velocities and measuring the velocities during the isovolumetric contraction period, and appears to provide additional information beyond two dimensional measurements [21]. In RV function assessment DTI is not significantly affected by volume loading and demonstrates a good reproducibility [22].

\section{Systolic myocardial velocity $(\mathrm{Sv})$}

In our study tricuspid annular (lateral) systolic velocities did not show significant change immediately after PTMC and is comparable to previous studies. Bensaid et al observed a non-significant increase 
Citation: Santosh Kumar C, Rajasekhar D, Vanajakshamma V, Boochi Babu M (2015) The Immediate and Short Term Impact of Successful Percutaneous Transvenous Mitral Commissurotomy on Right Ventricular Function. J Cardiovasc Dis Diagn 3: 217. doi:10.4172/23299517.1000217

Page 5 of 7

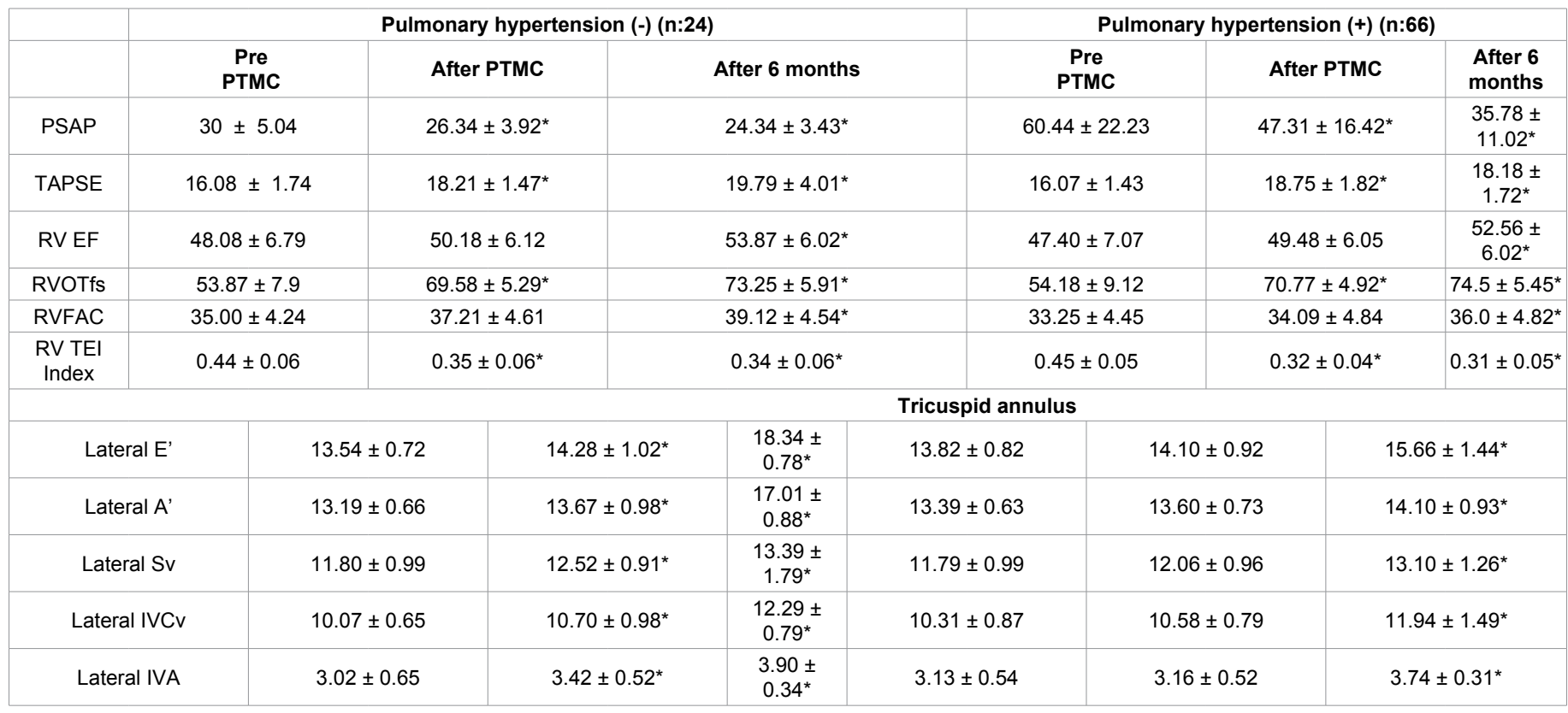

${ }^{*} \mathrm{P}<0.05$ comparison with baseline-after PMBV and 6 months

PTMC: Percutaneous transvenous mitral Commissurotomy; PASP: Pulmonary Artery Systolic Pressure; TAPSE: Tricuspid Annular Plane Systolic Excursion; RVEF: RV Ejection Fraction; RVOTfs: RV outflow Tract Fractional Shortening: RVFAC: RV Fractional Area Change; Ev: E wave velocity; Av: A wave velocity; Sv: Systolic Myocardial Velocity; IVCV: Myocardial velocity during isovolumic contraction; IVA: Isovolumetric Velocity Acceleration

Table 6: Comparison of pre- and post-PTMC (at 48 hours and 6 months) echocardiographic variables in patients with or without baseline pulmonary hypertension

in tricuspid annular Sv [23]. Drighil et al. found that Sv did not change immediately after PTMC [24]. In study done by Wang et al. tricuspid Sv was the best predictor of RV ejection fraction among several echocardiographic parameters [25]. Saxena et al. [26] noticed a strong correlation between tricuspid Sv and RV fractional area change, regardless of pulmonary artery pressures. On the other hand Ragab A et al. [27] noticed significant increase in tricuspid Sv after PTMC and also concluded that tricuspid Sv and TAPSE may precociously recognize patients with poor prognosis especially after PTMC. The absence of change in Sv immediately after PTMC may be due to less load dependence of this parameter.

On follow up at 6 months after PTMC we noticed a significant improvement in the tricuspid Sv suggesting that improvement in RV contractility occurs during follow up period after PTMC. This may be the result of positive RV remodeling after successful PTMC.

On subgroup analysis, tricuspid annular Sv improved significantly immediately after PTMC and continued to improve at 6 months in patients without $\mathrm{PAH}$. In patients with $\mathrm{PAH}, \mathrm{Sv}$ did not improved significantly immediately after PTMC and improved significantly at 6 months due to the positive RV remodeling after successful PTMC.

\section{Isovolumetric velocity acceleration (IVA)}

In our study we noticed no significant change in IVA measured at lateral tricuspid annulus immediately after PTMC comparable to other studies. At 6 months follow up there was significant improvement indicating improvement of RV function unrelated to after load reduction. On subgroup analysis, IVA improved significantly immediately after PTMC and continued to improve at 6 months in patients without PAH. In patients with PAH, IVA did not improved significantly immediately after PTMC but improved significantly at 6 months.
In an animal model, Vogel et al. [28] showed that IVA as a measure of RV contractility was not affected by preload within physiological ranges. IVA measures rate change of contractile force during isovolumic contraction; hence it was suggested as a strong index of RV contractility. Tayyareci et al. showed the reliability of RV IVA in the early detection of RV dysfunction in MS patients [29].

\section{Myocardial velocity during isovolumic contraction (IVCv)}

Our study showed that IVCv of lateral tricuspid annulus not changed significantly immediately after PTMC, but significant increase was noted at 6 months follow up comparable to previous studies [24,30]. Myocardial velocities of lateral tricuspid annulus and RV IVA were not changed immediately after PTMC because they are not affected by acute change in afterload after PTMC. But there was significant increase in these parameters at 6 months follow up study, suggesting that there is a true improvement in RV contractility over time after PTMC. On subgroup analysis, IVCv improved significantly immediately after PTMC and continued to improve at 6 months in patients without PAH. In patients with PAH, IVCv did not improved significantly immediately after PTMC but improved significantly at 6 months.

\section{Two-Dimensional echocardiographic assessment of RV function}

\section{a) RV TEI INDEX and RVOTfs}

Immediately after PTMC RV TEI index decreased significantly in both PAH and non PAH groups, comparable to previous studies. Drighil et al. noticed a significant improvement in RV TEI index from $0.5 \pm 0.2$ to $0.3 \pm 0.2(\mathrm{p}<0.0001)$ and Bensaid et al. observed a nonsignificant improvement in TEI index after PTMC from $0.33 \pm 0.1$ to $0.36 \pm 0.12(p=0.2) \quad[30,23]$. There was good correlation between TEI index and PASP before PTMC. These findings may mean an 
Citation: Santosh Kumar C, Rajasekhar D, Vanajakshamma V, Boochi Babu M (2015) The Immediate and Short Term Impact of Successful Percutaneous Transvenous Mitral Commissurotomy on Right Ventricular Function. J Cardiovasc Dis Diagn 3: 217. doi:10.4172/23299517.1000217

Page 6 of 7

improvement in RV contractility but may also reflect the acute fall in afterload.

The improvement in RV TEI index along with improvement in RVOTfs immediately after PTMC suggest an improvement in RV outflow tract systolic function as a result of acute decrease in RV afterload and not necessarily to improvement in RV contractility. In the study by Drighil et al. RVOTfs increased significantly from $57 \pm$ $15 \%$ to $72 \pm 12 \%, \mathrm{p}<0.0001$ [30]. When we studied at 6 months there was further improvement in both RV TEI index and RVOTfs may be due to further fall in RV afterload in both $\mathrm{PAH}$ and non $\mathrm{PAH}$ groups.

\section{b) RVEF and FAC}

Both RVEF and RVFAC did not change immediately after PTMC but improved significantly 6 months after PTMC in both PAH and non PAH groups. In the study by Drighil et al. there was a positive trend in the data of these parameters $(p=0.27$ and $p=0.24)$ [30]. Lack of significant improvement immediately after PTMC may reflect that these are measures of RV inflow and are afterload independent. In the study by Burger et al., he noticed that improvement in RVEF after PTMC depends directly on gain in stroke volume and inversely on RVEF before PTMC [9].

\section{c) TAPSE}

In our study TAPSE increased significantly from $16.0 \pm 1.5 \mathrm{~mm}$ to $18.6 \pm 1.7 \mathrm{~mm}, \mathrm{p}<0.0001$. This is comparable to previous study by Ragab et al. [27]. TAPSE increased significantly after PTMC from 17.1 \pm 2.1 to $19.1 \pm 2.5, \mathrm{p}<0.05$. On the other hand Bensaid et al. and Drighil et al. noticed a non-significant change in TAPSE after PTMC $[23,30]$. At 6 months follow up TAPSE improved further to $19.9 \pm 2.6 \mathrm{~mm}$ (p $<0.0001$, compared to both pre PTMC and immediate post PTMC values), suggesting there was gradual improvement in RV longitudinal function during follow up period after PTMC. On subgroup analysis TAPSE was significantly higher in both PAH and non PAH groups.

There was no significant change in left ventricle EF after PTMC from $60.0 \pm 5.8$ to $60.2 \pm 5.7, \mathrm{p}=0.2$. Similarly no change in LVEDD and LVESD was seen after PTMC implying that overall LV function was unchanged.

\section{Correlation between MVA and other parameters before PTMC}

We did not find any correlation between MVA by planimetry and DTI findings similar to the previous study [12].

\section{Limitations}

Patients with atrial fibrillation were excluded in the study; hence results cannot be generalized to all patients with MS. As controls were not included, magnitude of right ventricular dysfunction in patients with MS admitted for PTMC could not be compared to age and sex matched controls. In order to know the prognostic value of echocardiographic parameters of RV function after PTMC long term follow up with clinical variables is needed.

\section{Conclusions}

In patients with MS who underwent successful PTMC, there was immediate improvement in infundibular and global RV function as assessed by RVOTfs, TAPSE and RVTEI index. During follow-up period after PTMC there was gradual improvement in RV contractility as assessed by DTI. The myocardial velocities measured at lateral tricuspid annulus are afterload independent measures of RV systolic function, along with isovolumic velocity acceleration are useful for assessment of RV contractility in MS particularly in the early stages.

\section{Clinical implications of the present study}

The myocardial velocities measured at the lateral tricuspid annulus are afterload independent measures of RV systolic function, along with isovolumic velocity acceleration are useful for assessment of RV contractility in MS particularly in the early stages.

As improvement in RV contractility assessed by DTI less in PAH group when compared with non $\mathrm{PAH}$ group, MS patients should be intervened at an earlier stage before the development of PAH.

\section{References}

1. Carroll JD, Feldman T (1993) Percutaneous mitral balloon valvotomy and the new demographics of mitral stenosis. JAMA 270: 1731-1736.

2. lung B, Baron G, Butchart EG, Delahaye F, Gohlke-Bärwolf C, et al. (2003) A prospective survey of patients with valvular heart disease in Europe: The Euro Heart Survey on Valvular Heart Disease. Eur Heart J 24: 1231-1243.

3. Inoue K, Owaki T, Nakamura T, Kitamura F, Miyamoto N (1984) Clinica application of transvenous mitral commissurotomy by a new balloon catheter. $J$ Thorac Cardiovasc Surg 87: 394-402.

4. lung B, Garbarz E, Michaud P, Helou S, Farah B, et al. (1999) Late results of percutaneous mitral commissurotomy in a series of 1024 patients. Analysis of late clinical deterioration: frequency, anatomic findings, and predictive factors. Circulation 99: 3272-3278.

5. Vahanian A, Baumgartner H, Bax J, Butchart E, Dion R, et al. (2007) Guidelines on the management of valvular heart disease: The Task Force on the Management of Valvular Heart Disease of the European Society of Cardiology. Eur Heart J 28: 230-268.

6. Nishimura RA, Carabello BA, Faxon DP, Freed MD, Lytle BW, et al. (2008) ACC/AHA 2008 guideline update on valvular heart disease: focused update on infective endocarditis: a report of the American College of Cardiology/American Heart Association Task Force on Practice Guidelines: endorsed by the Society of Cardiovascular Anesthesiologists, Society for Cardiovascular Angiography and Interventions, and Society of Thoracic Surgeons. Circulation 118: 887-896.

7. Mohan JC, Sengupta PP, Arora R (1999) Immediate and delayed effects of successful percutaneous transvenous mitral commissurotomy on global right ventricular function in patients with isolated mitral stenosis. Int $\mathrm{J}$ Cardiol 68: 217-223.

8. Borer JS, Hochreiter C, Rosen S (1991) Right ventricular function in severe non-ischaemic mitral insufficiency. Eur Heart J 12 Suppl B: 22-25.

9. Burger W, Brinkies C, Illert S, Teupe C, KneissI GD, et al. (1997) Right ventricular function before and after percutaneous balloon mitral valvuloplasty Int J Cardiol 58: 7-15.

10. Hirata N, Sakakibara T, Shimazaki Y, Watanabe S, Nomura F, et al. (1992) Preoperative and postoperative right ventricular function during exercise in patients with mitral stenosis. J Thorac Cardiovasc Surg 104: 1029-1034.

11. Mohan JC, Sengupta PP, Arora R (1999) Immediate and delayed effects of successful percutaneous transvenous mitral commissurotomy on global right ventricular function in patients with isolated mitral stenosis. Int $\mathrm{J}$ Cardiol 68 : 217-223.

12. Ayman Morttada, Azza ElFiky, Ahmed Onsy, Sameh Samir,Gihan Toema (2015) Echocardiographic effect of successful balloon mitral valvuloplasty on right ventricular function. The Egyptian Heart Journal 67: 33-39.

13. Inci S, Erol MK, Bakırcı EM, Hamur H, Değirmenci H, et al. (2015) Effect of percutaneous mitral balloon valvuloplasty on right ventricular functions in mitral stenosis: short- and mid-term results. Anatol J Cardiol 15: 289-296.

14. Rudski LG, Lai WW, Afilalo J, Hua L, Handschumacher MD, et al. (2010) Guidelines for the echocardiographic assessment of the right heart in adults: a report from the American Society of Echocardiography endorsed by the European Association of Echocardiography, a registered branch of the European Society of Cardiology, and the Canadian Society of Echocardiography. J Am Soc Echocardiogr 23: 685-713. 
Citation: Santosh Kumar C, Rajasekhar D, Vanajakshamma V, Boochi Babu M (2015) The Immediate and Short Term Impact of Successful Percutaneous Transvenous Mitral Commissurotomy on Right Ventricular Function. J Cardiovasc Dis Diagn 3: 217. doi:10.4172/23299517.1000217

15. Ueti OM, Camargo EE, Ueti Ade A, de Lima-Filho EC, Nogueira EA (2002) Assessment of right ventricular function with Doppler echocardiographic indices derived from tricuspid annular motion: comparison with radionuclide angiography. Heart 88: 244-248.

16. Gaasch WH, Cole JS, Quinones MA, Alexander JK (1975) Dynamic determinants of letf ventricular diastolic pressure-volume relations in man. Circulation 51: 317-323

17. Anavekar NS, Gerson D, Skali H, Kwong RY, Yucel EK, et al. (2007) Twodimensional assessment of right ventricular function: an echocardiographicMRI correlative study. Echocardiography 24: 452-456.

18. Eidem BW, Tei C, O'Leary PW, Cetta F, Seward JB (1998) Nongeometric quantitative assessment of right and left ventricular function: myocardia performance index in normal children and patients with Ebstein anomaly. J Am Soc Echocardiogr 11: 849-856.

19. Lindqvist $P$, Waldenström A, Henein M, Mörner S, Kazzam E (2005) Regional and global right ventricular function in healthy individuals aged 20-90 years: a pulsed Doppler tissue imaging study: Umeå General Population Heart Study. Echocardiography 22: 305-314.

20. Duan YY, Harada K, Toyono M, Ishii H, Tamura M, et al. (2006) Effects of acute preload reduction on myocardial velocity during isovolumic contraction and myocardial acceleration in pediatric patients. Pediatr Cardiol 27: 32-36.

21. Meluzín J, Spinarová L, Bakala J, Toman J, Krejcí J, et al. (2001) Pulsed Doppler tissue imaging of the velocity of tricuspid annular systolic motion; a new, rapid, and non-invasive method of evaluating right ventricular systolic function. Eur Heart J 22: 340-348.

22. Kukulski T, Hübbert L, Arnold M, Wranne B, Hatle L, et al. (2000) Norma regional right ventricular function and its change with age: a Doppler myocardial imaging study. J Am Soc Echocardiogr 13: 194-204.

23. Bensaid A, Krapf L, Malanca M, Brochet E, lung B, et al. (2011)
Echocardiographic right ventricular function assessment before and after successful percutaneous mitral commissurotomy. J Heart Valve Dis 20: 627632.

24. Drighil A, Ghellab D, Mathewson JW, Ouarga L, Alalou H, et al. (2012) Immediate impact of successful percutaneous mitral valve commissurotomy on echocardiographic measures of right ventricular contractility. J Am Soc Echocardiogr 25: 1245-1250.

25. Wang J, Prakasa K, Bomma C, Tandri H, Dalal D, et al. (2007) Comparison of novel echocardiographic parameters of right ventricular function with ejection fraction by cardiac magnetic resonance. J Am Soc Echocardiogr 20: 10581064 .

26. Saxena N, Rajagopalan N, Edelman K, López-Candales A (2006) Tricuspid annular systolic velocity: a useful measurement in determining right ventricular systolic function regardless of pulmonary artery pressures. Echocardiography 23: $750-755$.

27. Ragab A, Mahfouz, Abdou M, Abdelmoneim A, Mostafa T (2011) Usefulness of non-invasive right ventricular function assessment in predictin of adverse events after successful balloon mitral valvuloplasty. J Basic Appl Sci Res 1: 260-268.

28. Vogel M, Schmidt MR, Kristiansen SB, Cheung M, White PA, et al. (2002) Validation of myocardial acceleration during isovolumic contraction as a novel noninvasive index of right ventricular contractility: comparison with ventricular pressure-volume relations in an animal model. Circulation 105: 1693-1699.

29. Tayyareci Y, Nisanci Y, Umman B, Oncul A, Yurdakul S, et al. (2008) Early detection of right ventricular systolic dysfunction by using myocardial acceleration during isovolumic contraction in patients with mitral stenosis. Eur J Echocardiogr 9: 516-521.

30. Drighil A, Bennis A, Mathewson JW, Lancelotti $P$, Rocha $P$ (2008) Immediate impact of successful percutaneous mitral valve commissurotomy on right ventricular function. Eur J Echocardiogr 9: 536-541. 